\title{
La brecha digital en las escuelas rurales: un estudio de caso
}

\section{The digital divide in rural schools: a case study}

José Sastre Reyes

Universidad de Murcia, España (josesastrereyes@gmail.com)

\section{RESUMEN:}

La presente investigación se centra en la problemática existente sobre la falta de recursos y formación en cuanto al uso de las tecnologías de la información y la comunicación (TIC), en la escuela rural andaluza. La investigación se realizó en el colegio público rural Alto Almanzora de la localidad almeriense de Armuña de Almanzora. La metodología utilizada fue un enfoque cualitativo que, según las características de esta investigación, fue necesario optar por la realización de un estudio de caso. La muestra se componía de un total de 15 alumnos y una maestra. Para la recogida de datos se planteó diseñar dos entrevistas semiestructuradas dirigidas por un lado al alumnado y otra para la maestra. Los resultados mostraron que el alumnado no cuenta con propuestas formativas específicas sobre el uso de las TIC, solamente en las asignaturas de Cultura digital y Ciudadanía. La maestra presenta un bajo nivel de competencia digital docente y no realiza formación específica sobre el uso de las TIC con el alumnado. Y, por último, el centro cuenta con recursos tecnológicos limitados al uso del alumnado de quinto y sexto, por lo que se encuentra una falta de recursos y formación en la mayoría de los niveles educativos de dicho centro.
PALABRAS CLAVE: ESCUELA RURAL, BRECHA DIGITAL, COMPETENCIA DIGITAL.

\begin{abstract}
:
The present research focuses on the existing problems regarding the lack of resources and training in the use of ICT in the Andalusian rural school. The research was carried out at Alto Almanzora, a rural public school located in Armuña de Almanzora, a town in Almería. The methodology used was a qualitative approach and, according to the characteristics of this research, it was necessary to opt for a case study. The sample consisted of a total of 15 students and a teacher. For data collection, two semi-structured interviews were planned, directed on the one hand to the students and on the other hand to the teacher. The results showed that the students do not benefit from specific training proposals on the use of ICT, only in the subjects of Digital Culture and Citizenship. The teacher has a low level of digital teaching competence and does not receive specific training on the use of ICT with students. And, finally, the technological resources at the school can only be used by fifth and sixth grade students, so a lack of resources and training is evident in most of the educational levels at the school.
\end{abstract}


KEYWORDS: RURAL SCHOOL, DIGITAL DIVIDE, DIGITAL COMPETENCE.

\section{INTRODUCCIÓN}

En las últimas décadas la escuela rural andaluza ha experimentado una serie de cambios a consecuencia de la introducción de las TIC. Raso, Trujillo y Ágreda (2015) señalan que el entorno rural se ha renovado en la actualidad en comparación al contexto rural que se databa en los orígenes de las escuelas rurales hace más de 20 años. También se ha hecho notable la evolución en los colegios rurales donde en sus inicios se contaba con infraestructuras deficitarias, que han dado paso a unas instituciones que gozan de modernas instalaciones donde se hace uso de las TIC. Pero a pesar de contar con estas instalaciones, se destaca el retraso con el que la administración ha puesto en marcha los diferentes programas para la integración de las TIC en los centros rurales, en comparación con otros centros educativos.

Este tipo de problemas relacionados con la falta de dotación tecnológica se le denomina brecha digital. Autores como Juliá, García y Meliá (2006) señalan que la brecha digital supone un obstáculo para el desarrollo rural. La mayoría de la problemática deriva del acceso a los servicios de Internet, independientemente de la zona geográfica donde habitan. En este sentido, la brecha digital en el medio rural se limitaría a observar si los usuarios tienen acceso a: la disponibilidad de un ordenador, acceso a la red desde el trabajo, el hogar, etc., el conocimiento sobre el uso de las herramientas básicas que facilitan el acceso a Internet y, por último, la capacidad del usuario para gestionar la información de la red en conocimiento.

Para poder llevar a cabo esta mejora de la brecha digital se debe desarrollar el conocimiento sobre el uso de estas herramientas en los usuarios, a lo que se le conoce como competencia digital. Moral y Villalustre (2011) señalan que el perfil del profesorado se ha acondicionado a la integración de las herramientas digitales en los procesos de enseñanza y aprendizaje. Esto exige una actualización permanente de los conocimientos del profesorado en relación con las TIC con el fin de renovar la educación en el medio rural, lo que requerirá de la creación de comunidades de práctica que aseguren el desarrollo de la competencia digital en todos los miembros de la escuela rural.

Con la presente investigación se pretende conocer el uso de las TIC dentro de la escuela rural, concretamente en el alumnado de Educación Primaria. En este caso, nos centramos en describir los hábitos en cuanto al manejo de las TIC que realizan la docente y los alumnos en este contexto rural. También se detallará el proceso formativo con las TIC, a la vez que los recursos con los que trabaja la comunidad educativa. Los participantes que forman esta investigación son los alumnos de cuarto, quinto y sexto del colegio público rural Alto Almanzora, situado en la localidad de Armuña de Almanzora de Almería. En este caso la muestra la forman los alumnos y la maestra responsable del aula multigrado de dicho centro.

Los objetivos principales de este estudio se centran en describir las prácticas educativas con TIC que hace la maestra de un colegio rural, conocer el uso educativo de las TIC de los alumnos de un colegio rural y detectar las posibles necesidades sobre el uso educativo de las TIC en el colegio rural. Para la recogida de datos se determina la realización de dos entrevistas semiestructuradas dirigidas por un lado a la maestra y por otro al alumnado.

\section{MÉTODO}

\subsection{Preguntas de investigación}

El problema de esta investigación se ha centrado en conocer si existen dificultades sobre el uso de las TIC en los alumnos de un colegio rural de Almería. Este centro cuenta con niños de edades comprendidas entre los 10 y 12 años. La brecha digital se ha hecho evidente en el medio rural debido a la falta de recursos tecnológicos o de formación. Este déficit en la formación de los usuarios puede limitar el desarrollo de la competencia digital. En este sentido, la 
problemática se centraría en conocer los procesos formativos que implican el uso de las TIC por parte de este alumnado, con el fin de detectar posibles dificultades para crear contenido digital (texto, tablas, imágenes, archivos de audio, etc.). También sería conveniente describir los recursos con los que cuenta el centro, para ver si se detecta algún tipo de brecha digital en este entorno.

Partiendo como punto de partida de la problemática existente es el momento de formular las hipótesis con las que se va a trabajar en esta investigación.

En este caso, las hipótesis quedan definidas de la siguiente forma:

- La falta de formación de los docentes limita el desarrollo de la competencia digital de los niños en el medio rural.

- La falta de recursos limita el uso seguro de internet de los adolescentes en el medio rural.

A raíz de las hipótesis presentadas anteriormente se han extraído las variables con las van a trabajar:

- Búsqueda de información.

- El uso seguro de internet.

- La falta de formación.

- La falta de recursos.

\subsection{Objetivos}

Los objetivos que pretende alcanzar el presente trabajo serían los siguientes:

1. Describir las prácticas educativas con TIC que hace una maestra de colegio rural.

1.1. Detectar las posibles dificultades qué tiene la maestra para utilizar las tecnologías en su práctica docente.

1.2. Conocer la formación en competencia digital de la maestra de un colegio rural.

2. Conocer el uso educativo de las TIC de los alumnos de un colegio rural.

2.1. Conocer los recursos tecnológicos en un colegio rural.

2.2. Conocer la formación de los alumnos en el uso de las TIC.
3. Detectar las posibles necesidades sobre el uso educativo de las TIC en el colegio rural.

3.1. Identificar las necesidades formativas sobre el uso educativo de las TIC en el colegio rural.

3.2. Detectar las necesidades tecnológicas que presenta el colegio rural.

\subsection{Método}

El paradigma de esta investigación es de carácter interpretativo. Para describir las características de este paradigma vamos a tomar como referencia a Reeves (2006), quien establece este tipo de clasificación y lo define como una realidad construida por la sociedad en la que se intenta interpretar los fenómenos, por lo que la posición del investigador sería de inmersión, ya que, en este caso, se encontraría dentro del centro educativo teniendo una toma de contacto con los individuos para poder interpretar los fenómenos desde la realidad de los sujetos.

Esta investigación es cualitativa ya que sería un estudio de caso, donde MacMillan y Schumacher (2007) lo definen como una investigación que pretende conocer la mayor cantidad de aspectos posibles sobre una situación concreta. Todos estos aspectos se sistematizan para poder comprender la complejidad de este proceso.

\subsection{Contexto}

Esta investigación se lleva a cabo en el colegio público rural Alto Almanzora de Armuña de Almanzora en la provincia de Almería. La sede de dicho Centro se encuentra en la localidad de Armuña de Almanzora. Los maestros tienen que itinerar entre varias localidades para satisfacer la demanda de cada una de las sedes. La itinerancia la suelen realizar los maestros especialistas con una media de 3 y 4 localidades. Por el contrario, los tutores suelen dar clase en la sede donde se ubica su clase, evitando itinerar por la gran carga lectiva de horas que tienen en este caso.

\subsection{Participantes}


El colegio se compone de 130 alumnos y 18 maestros. Los participantes en el proceso de recogida de datos son los siguientes:

- 15 alumnos de cuarto, quinto y sexto curso.

- La maestra correspondiente a dicha clase.

\subsection{Instrumento}

El instrumento seleccionado para la recogida de datos ha sido la entrevista semiestructurada. De manera que ha sido necesario diseñar dos entrevistas diferentes: una para utilizarla con la maestra y la segunda con el alumnado. Ha sido necesario desplazarse hasta el colegio para la recogida de datos ante la imposibilidad de realizarla a distancia.

En ambas entrevistas se diseñaron las preguntas teniendo en cuenta una serie de variables que se detallan a continuación:

- Falta de formación.

- Falta de recursos.

Enlace a las entrevistas: http://cort.as/-LdgP

\subsection{Análisis de datos y tratamiento de la información}

El análisis de datos se realizó a partir de las transcripciones realizadas de los distintos audios obtenidos en la recogida de datos. Para ello se utilizó el software de análisis de datos cualitativos atlas.ti. Se procedió a analizar los datos obteniendo las distintas citas en cada una de las entrevistas, que posteriormente fueron codificadas en diferentes códigos asignados por bloques. Una vez codificada toda la información se creó una red semántica estableciendo las relaciones correspondientes entre los distintos códigos.

\section{RESULTADOS}

Se ha realizado un análisis de datos de tipo cualitativo, porque los resultados se han interpretado utilizando las citas textuales de las entrevistas, por lo que se ha tenido en cuenta las relaciones que se dan entre las respuestas y las citas que se repetían con mayor frecuencia.

Los resultados se han dividido en tres bloques que comprenden: el uso de las TIC por el alumnado, el uso de las TIC por parte de la maestra y, por último, los recursos de la escuela rural.

Antes de nada, vamos a ver el porcentaje de alumnos en relación con cada curso en la clase. Encontramos que un $33 \%$ de la clase se corresponde con cuarto curso, el $20 \%$ con quinto de primaria y el $47 \%$ con sexto curso.

\subsection{Uso que los alumnos hacen de las TIC}

En primer lugar, vamos a describir cuáles son los dispositivos que utiliza el alumnado en clase. El alumnado de sexto afirma utilizar los siguientes dispositivos: la pizarra digital y los ordenadores; el alumnado de quinto dice que hace uso de: la pizarra digital y los ordenadores. Entre el alumnado de cuarto curso encontramos dos datos reveladores, la mayoría afirma que solo utiliza la pizarra digital y un alumno dice que no ha utilizado ningún dispositivo digital.

A continuación, se establece la relación entre los dispositivos utilizados por el alumnado y las asignaturas en las que se usan. El tema que más se repite entre el alumnado de sexto es que utilizan los ordenadores en Cultura digital; en los alumnos de quinto encontramos que lo utilizan en Ciudadanía y en el alumnado de cuarto destaca que utilizan la pizarra digital en todas las asignaturas y un alumno afirma que no usa el ordenador en ninguna asignatura.

También es importante señalar la frecuencia con la que los alumnos usan estas herramientas en clase. Entre el alumnado que utiliza los ordenadores encontramos que la mayoría del alumnado coincide en que lo utilizan dos o tres veces por semana. También observamos dos datos curiosos con respecto al alumnado que no utiliza ningún dispositivo digital en ninguna asignatura y es que solo utilizan los ordenadores en Cultura digital y, por otro lado, afirman utilizar la pizarra digital pocas veces, solo para corregir.

En cuanto a la formación recibida por la maestra sobre el uso de los ordenadores, el 
alumnado de sexto coincide en que reciben formación solamente en la asignatura de Cultura digital, el alumnado de quinto solo en Ciudadanía. Sin embargo, la mayoría del alumnado de cuarto curso afirma "no me han explicado nunca".

Por otro lado, se ha entrevistado al alumnado sobre el uso que hacen de Internet a la hora de preparar los trabajos de clase. En esta pregunta hemos encontrado que el tema más repetido es "para buscar información"; otro alumno afirma que "para traducir palabras y ver alguna película" y otro comenta que "para buscar cosas que me manda la maestra o algo que no sé".

A continuación, la investigación se centró en conocer los dispositivos más utilizados por el alumnado fuera del horario escolar. La opción más repetida entre el alumnado es "el móvil y el ordenador" seguido de "el ordenador y la tableta" y de "el móvil y la tableta". Como dato significativo encontramos que la mayor parte de los alumnos afirma utilizar el móvil en primer lugar, antes que otro tipo de dispositivos.

Habiendo analizado los datos sobre los dispositivos usados en casa, se decide obtener información sobre los hábitos de uso de dichas herramientas. El comentario mas repetido entre los alumnos es "para jugar a juegos"; otros estudiantes comentan que "para ver películas"; otro dato relevante es que los alumnos utilizan estos dispositivos "para buscar información" y el comentario menos repetido entre los estudiantes es que lo utilizan "para si algo no sé en las actividades, verlas".

Por último, se decidió entrevistar al alumnado para recoger información sobre los equipos tecnológicos que tiene el alumnado en casa. En este caso, el alumnado afirma tener los siguientes dispositivos en casa: "móvil y ordenador" y otro comentario menos repetido entre el alumnado dice que "tableta".

\subsection{Uso de las TIC por parte de la maestra}

Primeramente, nos centramos en conocer los equipos tecnológicos utilizados en clase $\mathrm{y}$ señala que la pizarra digital en todas las asignaturas para realizar correcciones, también 7 ordenadores portátiles que son utilizados por el alumnado en las asignaturas de Ciudadanía y Cultura digital.

En cuanto a su conocimiento sobre aplicaciones web dice no conocer ningún tipo de aplicación y, por tanto, no las utiliza en el aula. Solo usa las que ofrece el sistema operativo Guadalinex por defecto, como por ejemplo OpenOffice.

Dice que los problemas educativos los resuelve con la ayuda de las TIC, como pueden ser la necesidad de buscar información con el alumnado y el uso de la aplicación de presentaciones para elaborar los trabajos.

Sobre su formación sobre competencia digital docente, encontramos que sus últimos cursos realizados al respecto datan del año 2004/05 y con respecto a la introducción de la pizarra digital, pero posteriormente no ha realizado ningún tipo de formación al respecto.

Por otro lado, la maestra indica no haber realizado ningún tipo de acción formativa sobre el uso de las TIC con su alumnado, solamente alguna aproximación teórica.

Con respecto a las dificultades formativas para incluir las TIC en la práctica docente, indica que no todo el alumnado dispone de ordenador en casa, por lo que a su uso se refiere en el contexto escolar, aparecen diferentes niveles de aprendizaje, por lo que debe iniciar a este alumnado con más bajo conocimiento en el uso de los mismos, pero al ser muy jóvenes destaca que aprenden de una forma muy rápida.

\subsection{Los recursos TIC de la escuela rural}

En primer lugar, comparando los resultados de las entrevistas del alumnado y la maestra, encontramos que la escuela cuenta con un ordenador por cada docente, ordenadores y una pizarra digital que se usan con los estudiantes de quinto y sexto curso. Encontramos que el alumnado de la etapa de infantil y primero, segundo, tercer y cuarto curso de primaria no tienen acceso a ningún recurso tecnológico.

En cuanto a los aciertos del centro sobre la adopción de las TIC se destaca el aumento de la motivación en el alumnado y las distintas posibilidades que ofrecen a los docentes. Pero se argumenta que los errores que se 
encontraron, fue dotar al alumnado de ordenadores que nunca retornaron al centro, por lo que se produjo una mala gestión de los recursos por parte de la administración.

Un dato interesante fue la recogida de datos entre el alumnado para conocer las propuestas formativas que ofrece el centro al alumnado en relación a las TIC. Encontramos tres respuestas entre el alumnado: dos de ellas representaban a la mayoría de la clase y coinciden en la siguiente afirmación: "solamente la asignatura de competencia digital y el año pasado la asignatura de ciudadanía", correspondiéndose estas asignaturas con los niveles de quinto $\mathrm{y}$ sexto donde se imparten y otro grupo de la clase añade que "nunca he recibido alguna explicación, solo una vez. No tengo ninguna asignatura o curso" y se corresponde con el alumnado de cuarto curso. Por otro lado, la propuesta formativa para mejorar la competencia digital docente en el centro según la maestra es que disponen de un coordinador TIC que se encarga de resolver dudas y dificultades de tipo técnico. También disponen de los cursos de formación impartidos por el centro de profesorado, pero estos se realizan según la demanda docente.

\section{DISCUSIÓN}

A lo largo de este apartado vamos a contrastar los principales hallazgos de esta investigación con respecto al tema de estudio. Para ello vemos que Moya (2013) explica la importancia de crear contenidos educativos digitales para mejorar la competencia digital del alumnado, pero esto no era posible si no se mejora la competencia digital docente, todo ello con el fin de disminuir la brecha digital existente entre los docentes y los estudiantes. De manera que los resultados de este estudio indican que esto no ha sido posible en la actualidad, ya que encontramos a una docente que tiene una falta de formación en competencia digital docente. En este caso, vemos que la única formación que posee coincide con la implementación de las TIC en los centros educativos hace una serie de años $\mathrm{y}$, por consiguiente, no se ha realizado ningún tipo de curso para mejorar la competencia digital docente.

En este sentido, del Moral, Villalustre y Neira (2014) describen que Internet y las pizarras digitales han sido pilares para la innovación en busca del desarrollo de la competencia digital. En este trabajo, hemos encontrado que la incorporación de las TIC en la escuela rural ha supuesto un incremento en la motivación del alumnado, ya que le permite interactuar con nuevos contenidos digitales. Aún así, hemos visto que el alumnado no tiene acceso al uso de la pizarra digital porque la docente limita su uso a la corrección de actividades o como medio de apoyo en sus explicaciones, sin encontrar uso alguno de esta herramienta por parte del alumnado.

Se ha detectado la mala práctica docente en el uso de las TIC, lo que se asemeja al estudio elaborado por Raso, Hinojo y Solá (2014), donde se describen las limitaciones que encuentran los estudiantes en la aplicación y uso de estas herramientas digitales por consecuencia de la falta de formación o malas prácticas pedagógicas de los docentes. En nuestro caso, vemos que la docente a pesar de disponer de pizarra digital no facilita el acceso a los alumnos, con el fin de facilitar la realización de diferentes actividades de forma interactiva.

Ortega (2009) señala como factor determinante de cambio, la mejora de la alfabetización tecnológica centrada en los procesos formativos que contribuyen a reducir las desigualdades. Pero eso no ha sido así, vemos que en la escuela rural el alumnado no dispone de ningún tipo de formación específica por parte del centro para mejorar su competencia digital. Encontramos que la única formación se limitaba al uso de ordenadores en la asignatura de Cultura digital en sexto y Ciudadanía en quinto.

Esta falta de formación en el alumnado trasciende a raíz de la práctica docente y de una falta de propuestas del centro destinadas a mejorar tal fin. La importancia de tener un primer acercamiento a las TIC con un carácter formativo para reducir la brecha digital del alumnado con respecto a su formación. Un hallazgo en esta investigación nos permitió 
conocer que sigue existiendo la brecha digital en centros educativos como puede ser en la escuela rural. El resultado fue que el acceso al uso de los ordenadores se limitaba al alumnado del último ciclo, en relación a la poca disponibilidad de equipos en el colegio y la falta de utilización de los mismos en las áreas nombradas anteriormente. Esta desigualdad en el acceso a los ordenadores a todo el alumnado de la escuela rural, viene a raíz de una mala gestión de los recursos por parte del centro, al limitar su uso a una parte de los estudiantes y, por otra parte, a la poca disponibilidad de ordenadores para el alumnado.

\section{CONCLUSIONES}

El marco teórico de esta investigación se comprendía en tres bloques temáticos que eran la escuela rural, la brecha digital en el entorno rural $\mathrm{y}$, por último, el desarrollo de la competencia digital en el medio rural. En este sentido, se han descubierto una serie de hallazgos en esta investigación, con respecto a la información recogida en cada uno de los puntos anteriores. Encontramos que hay una mayor participación de los padres en la formación de sus hijos con respecto al uso de las TIC.

Los hallazgos sobre el nivel de competencia digital docente indican que no se encuentra iniciativa docente para mejorar los conocimientos, ya que la última formación recibida data del año 2005. Por otro lado, se mejoran las prácticas educativas al introducir formación sobre el uso de las TIC en las asignaturas de cultura digital y ciudadanía.

Por último, la brecha digital en el medio rural sigue siendo evidente. En el centro educativo de esta investigación encontramos que se dispone de recursos digitales, pero solamente tienen acceso el alumnado de los dos últimos cursos de primaria porque hay escasez de equipos informáticos para todo el centro y también se observa una mala gestión de los recursos por parte del mismo.

Por una parte, encontramos que la maestra no tiene un nivel adecuado de competencia digital docente, ya que señala que desde el año 2004/05 no ha realizado ningún tipo de formación sobre el uso de las TIC. En este sentido nos encontramos que no posee ningún tipo de conocimiento sobre las aplicaciones web; por lo tanto, no puede aplicarlo en su práctica educativa. En su labor docente no realiza ningún tipo de acción formativa con el alumnado, solamente dentro de las áreas de Cultura digital y Ciudadanía en quinto y sexto curso, pero como dato significativo encontramos que el alumnado de cuarto curso no utiliza las TIC a pesar de pertenecer a una clase que dispone de los dispositivos necesarios.

Por otro parte, la falta de recursos en este centro es evidente porque se dispone de un número limitado de ordenadores destinado al uso de los alumnos de quinto y sexto, por lo que el resto de niveles educativos carecen de acceso al uso de estos dispositivos informáticos. De igual manera también se aprecia la falta de programas formativos para ayudar al alumnado a aprender a usar estos dispositivos digitales. En este sentido, el profesorado tampoco participa en la realización de cursos formativos a pesar de disponer de una oferta permanente de cursos impartidos por el centro del profesorado.

A continuación, vamos a ver cuáles ha sido las principales limitaciones que ha presentado este estudio.

La falta de acceso a los datos supuso una limitación, ya que el colegio rural al estar diseminado en varias localidades presenta una dificultad a la hora de recoger datos de los diferentes maestros y alumnos, de ahí que se decidiera entrevistar al alumnado y la maestra de una clase, porque la recogida de datos presentaba dificultades de tiempo y acceso a todas las localidades.

Las limitaciones que encuentra el investigador son otro punto a tratar $\mathrm{y}$ encontramos las siguientes:

- El investigador encontró dificultades de acceso en cuanto a la recogida de datos porque el colegio no disponía de buenas conexiones de internet para poder realizar las entrevistas por videoconferencia, lo que condicionó al investigador y tuvo que visitar el colegio para la realización de las mismas. 
- A efectos longitudinales, encontramos que el tiempo para investigar el problema estuvo limitado en cierta manera, debido a la fecha de vencimiento de la entrega del proyecto.

- La inclusión en el estudio de una serie de propuestas prácticas para dar solución a las necesidades detectadas.

Mediante la realización de la presente investigación se han derivado posibles líneas de investigación:

- Habría que centrar la atención en descubrir nuevas formas gestionar los recursos en las escuelas rurales, para hacer frente a la escasez de equipos de los que se disponen.

- La falta de formación sería otra línea e iría encaminada a conocer si los docentes de la escuela rural han mejorado su nivel de competencia digital y descubrir las dificultades que limitan a estos docentes llevar a cabo este fin. Al igual que también se debería de indagar en las posibles mejoras que permitan desarrollar la competencia digital en el alumnado.

- También otra línea de investigación estaría centrada en conocer si las situaciones comentadas anteriormente, se producen en otras escuelas rurales.

- Este trabajo plantea la realización de distintas propuestas prácticas, con el objetivo de poder dar respuesta a las necesidades que hemos encontrado a lo largo de esta investigación.

\section{REFERENCIAS}

Juliá, J., García, G., y Meliá, E. (2006). Internet, cooperativismo agrario y desarrollo rural. El caso de las cooperativas de la Comunidad Valenciana. CIRIEC-España, Revista de Economía Pública, Social y Cooperativa, 55, 221-251.

Macmillan, J.H. y Schumacher, S. (2007). Investigación educativa: una introducción conceptual. PearsonAddison Wesley.

Moral, M. E. Del, Villalustre, L., y Neira, M. D. R. (2014). Oportunidades de las TIC para la innovación educativa en las escuelas rurales de Asturias. Aula Abierta, 42(1), 61-67. doi: 10.1016/S0210-2773(14)70010-1

Moral, E., y Villalustre, L. (2011). Las comunidades de práctica en la web 2.0 para la colaboración entre escuelas rurales. DIM: Didáctica, Innovación y Multimedia, 20, 1-8. Recuperado de http://www.raco.cat/index.php/DIM/article/viewArticle/ 243446/0

Moral, M. E. Del, Villalustre, L., y Neira, M. D. (2014). Oportunidades de las TIC para la innovación educativa en las escuelas rurales de Asturias. Aula Abierta, 42(1), 61-67. doi: 10.1016/S0210-2773(14)70010-1

Moya, M. (2013). De las TICs a las TACs: la importancia de crear contenidos educativos digitales. Revista Didática, Innovación Y Multimedia, 27, 1-15.

Ortega, I. (2009). La alfabetización tecnológica. Teoría de la Educación. Educación y Cultura en la Sociedad de la Información, 10(2), 11-24.

Raso, F., Hinojo, M. y Solá, J. (2014). Integración y uso docente de las tecnologías de la información y la comunicación (TIC) en la escuela rural de la provincia de Granada: estudio descriptivo. REICE. Revista Iberoamericana sobre Calidad, Eficacia y Cambio en Educación, 13(1), 139-159.

Raso, F., Trujillo, J. y Ágreda, M. (2015). Análisis de la integración curricular y la utilidad didáctica de las tecnologías de la información y la comunicación (TIC) en los colegios públicos rurales de Andalucía. Educational Research with social impact.

Reeves, T. C. (2006). Design research from the technology perspective. In J. van den Akker, K. Gravemeijer, S. Mckenney, y N. Nieveen (eds.), Educational design research (pp. 86-109). London: Routledge. 